Onsct of Cholecystitis.-By the end of the eighth week the patient was considered about fit for discharge, when suddenly, on February $23 r d$, she felt an acute pain on the right side of the abdomen and vomited about a pint of thick yellow fluid. As the pain was very severe, she was given half a grain of morphine hypodermically in the forenoon, and this was repeated again in the evening, as the pain was again beginning to trouble her. While the pain was severe she perspired freely. Her temperature was subnormal at first, but in the evening rose to $101^{\circ} \mathrm{F}$.; there was dullness, tenderness, and resistance on the right side in the region of the gall bladder, the dullness being continuous with the liver dullness, and extending down to the level of the umbilicus. Hot laudanum stupes were applied continuously umbilicis. Hot laudanum and during the night.

all day and during the night. On the following day the temperature was down to $100^{\circ} \mathrm{F}$. She had still a good deal of pain and was sick, but did not vomit. On account of the nature of the pain, the faeces were condition continued until March 19th, there being severe pain almost daily, which had to be cor trolled with morphine. The temperature was very irregular, at times being subnormal, when the paroxysms of pain were very severe, then, after a feeling of cold and shivering, rising to $102^{\circ}$ or $103^{\circ} \mathrm{F}$., and on one occasion to $105^{\circ} \mathrm{F}$. She was inclined to be sick and did not take nourishment well and lost flesh, though the swelling seemed to be gradually getting less-indeed, the lower margin on the enlarged gall bladder was now 2 in. higher than on February 24 th and 25 th.

Operation.-As she seemed to be losing ground and suffering much pain, it was deemed, after consultation with Dr. J. T. much pain, it was deemed, after consultation with Dr. J. T. the gall bladder, and the operation was performed by Dr. Peter the gall bladder, and the operation was performed by Dr. Peter Paterson, of Glasgow, on March 20th, who opened the gall bladder by a vertical incision through the right rectus muscle.
The gall bladder, which was small, firmly adhered to the colon, while its walls were thick and very friable. On opening it, an ounce of thick ochre-like pus escaped, and two gall stones abou the size of peas were removed. Drainage was provided by large rubber tube stitched to the incision in the gall bladder.

Result. - The bile drained away freely at first, but gradually decreased to $1 \mathrm{oz}$. in the day, upon which the tube was removed. When the tube was removed, on April 2nd, six rounded, rough concretions were found, which were very friable, two being broken in being removed. The further history of the case was aneventful, and on May 24th she was dismissed from hospital feeling fit and well, the wound having healed perfectly. The child was healthy and continued to thrive. The blood when child was healthy and continued to thrive. The blo

The Fluid from the Gall Bladder.-Dr. J. Hume Paterson, who examined specimens from the case at the County Bacteriologica Laboratory, furnished reports which may be summarized as follows :

Specimen No. 1 was a mixture of blood and purulent yellow material taken at the time of the operation. Smears prepare from this material and cultures on ordinary agar and McConkey's bile-salt agar plates showed an organism in great numbers that morphologically and culturally was identical "with Bacillus typhosus. As this was a mixed specimen containing blood from the wound as well as fluid from the gall bladder, and as ther was hope of obtaining further specimens, the orsanism found was not submitted to differential tests with the various carbohydrates, etc., but the patient's blood gave an agglutinating rea tion.

specimen No. 2 was bile fluid draining from the gall bladder. Smears and cultures showed an organism in great number that morphologically and culturally was identical with Bacillus typhosus. The usual subcultures were made with colonies from the ordinary agar plate, with the result that the organism was found to be Bacillus typhosus. Thus a culture in taurocholate broth gave with glucose a slightly acid reaction; with lactose saccharose, and dulcite, no acidity; with mannite, slight acidity; litmus milk gave a slight acidity, but no coagulation. 'The patient's blood gave an agglutinating reaction.

Specimen No. 3 was gall stones (5 in number, with several fragments). The whole stones were each about the size of an ordinary pea, pale yellow in colour, with nodular glistening surface. They were readily broken down, being of very soft consistence. Dissolved in hot absolute alcohol, allowed to recrystallize, and examined microscopically, they were found to be composed of cholesterin crystals and mucin. Two of the stones were washed well in absolnte alcohol, and then in sterile stones were washed when in sterile water. They were then split open with a sterile knife, and atained and examined for micro-organisms with negative results.

'The more important points in this case might be summarized thus:

1. 'The presence of almost continuous constipation throughout the whole course of an ordinary typhoid illness.

2. The development of inflammation of the gall bladder during convalescence, about the eighth week of illness.

3. The development of peritonitis in connexion therewith, forming adhesions between the gall bladder and the colon.

4. The presence of Bacillus typhosus in pure culture, and

5. The presence of a few gall stones, consisting of cholesterin and mucin:
In conclusion, I wish to express my thanks to Dr. Peter Paterson, the Surgeon, and Dr. J. Hume Paterson, the Bacteriologist, who were associated with me in dealing with the case. Also to Dr. J. T. Wilson, the Medical Officer of Health for Lanarkshire, for much helpful advice, and for permission to publish my notes.

\section{A CASE OF FIBROMYOMA OF THE STOMACH.}

By DOUGLAS GORDON CHEYNE, M.B., Ch.B., RESIDENT MEDICAL OFFICER, ROYAL WATERLOO HOSPITAL.

THE rarity of the case, of which I here give a few notes, is my excuse for reporting it; taken in conjunction with the case which Mr. W. T. Green, F.R.C.S., published in -the British Medical Journal of October 28th, 1911, its interest is increased

Though resembling Mr. Green's case in general, in detail it was quite different and also wholly unlike any of the other older cases, notes of which were published in the same article.

In the present case the patient appeared almost unaware of the presence of the tumour and made no complaints

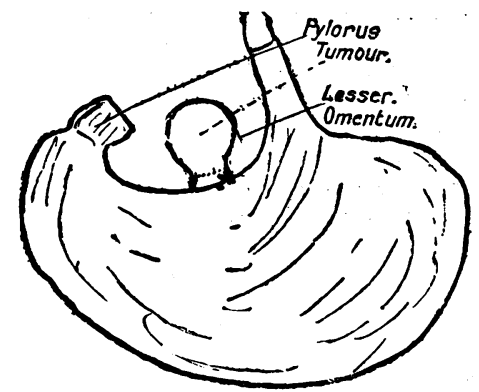

therefore one may presume there were no symptoms. Indeed she sought advice in our out-patient department for an entirely different complaint, namely, prolapsus uteri, and it was only by the merest chance that the tumour was discovered at all. In the out-patient department the signs were suggestive of a subperitoneal fibroid detached, or an ovarian cyst with a long pedicle. It was at that time thought that the mass was movable in all directions, but subsequently this proved to be incorrect. She was accordingly admitted into our wards as a gynaecological case.

The patient was a married woman, aged 59, who had had three children and three miscarriages. She had been subject to bleeding from the rectum after defaecation for mony years, and had been wearing a ring pessary on account of a prolapsed uterus. The menopause had occurred twelve years before. About a year ago she noticed a small lump in the abdomen, and fancied it had been getting bigger, but it had caused no symptom.

Condition on Admassion. - She was a lealthy, rather obese woman, with an excellent colour. Had no complaints. Teeth in good condition, tongue clean, appetite good. $B^{n}$ wels regular. Temperature $98^{\circ}$, pulse 80 , respirations 25 . In the epigastrium, in the middle line, is seen a distinct bulging, which appears to move a little upwards and downwards on respiration on palpation a firm, smooth, rounded, elastic mass, about the size of a cricket boll, is felt. It permits of consider, about the size in a considerable movement in a lateral direction, being capable of being drawn over to either lumbar fossae, but does not allow movement in a vertical direc'tion below the level of the umbilicus. It has evidently no con-
nexion with the pelvis. The note obtained on percussion is nexion with the pelvis. The note obtained on percussion is absolutely flat. Auscultation of the stomach reveals no evidence of dilatation of that organ. Vaginal examination
revealed signs of rectocele and cystocele, but otherwise nothing abnormal is found. There were well-marked external haemor rhoids, now in an inflamed condition, which probably accounts for the bleeding on defaecation. The urine was acid, specific gravity 1022 ; no albumen or sugar ; some phosphates.

Diagnosis.-The case was seen soon after admission by $\mathrm{Dr}$. Gow, who decided that, apart from the prolapse, it was not a gynaecological case, and referred it to the surgical side, where she was examined by Mr. Pendlebury, Dr. J. D. Mortimer and myself being present. On that occasion no trace of the tumour which before had been so obvious, could be found. The next day it reappeared exactly as before, and was again seen by Mr. Pendlebury, who thought it might possibly be an omental Mr. Pendlebury, who thought it might possibly be an omental
cyst, or a pyloric tumour, and decided, subject to the approval cyst, or a pyloric tumour, and decided, subject to the approval
of the patient and her friends, to do an exploratory operation. of the patient and her friends, to do an exploratory operation.
This was readily given, and an anaesthetic was administered on This was readily given, and an anaes
October $28 \mathrm{th}$ by Dr. J. D. Mortimer. 
Operation.-An incision was made through the rectus muscles in the right hypochondriac region, and the peritoneal cavity opened. The tumour at once presented itself, and was about the size of a cricket ball, and gro wing from the lesser curvature of the stomach, between the layer of the lesser omentum. The rough sketch given may better explain this. There were no adhesions. There was a short pedicle along which no a the vols came the vessels supplying it, arising from the edge of the lesser curvature. An incision was made through the peritoneal surface round the base, and after ligaturing the pedicle the tumour was easily removed by blunt dissection. There was practically no haemorrhage. The cut peritoneal surfaces were united by Lembert sutures. The stomach and other abdominal viscera appeared to be perfectly normal. The abdominal wall was closed in layers. The operation lasted fifteen minutes. There was very little sickness or discomfort af terwards.

Resiult.-The convalescence was uneventful. Stitches were removed on the eleventh day, and the patient got up on the eighteenth day, and went home on the twentieth day.

The tumour excised weighed $4 \mathrm{oz}$. Its surface was smooth. It was softish in consistency, and at places almost cystic. The pathological report was as follows:

The tumour has the structure of a fibromyoma, composed of interlacing bundles of smooth muscle cells enclosed in a scanty stroma. It shows some areas of mucoid degeneration, and is in parts infiltrated with leucocytes. At one end of the tumour the appearance to the naked eve suggests the red degeneration so often seen in uterine fibromyoma. It appears to be an encapsuled growth, and is not malignant.

In conclusion, the disappearance of the tumour may be explained by its having been drawn underneath the liver while the stomach was very empty. Its origin, its lack of symptom, and its structure all combine to show horv difficult may be the diagnosis of these cases, a difficulty which can only be overcome by an exploratory operation.

I wish to express to Mr. Pendlebury my best thanks for his kind permission to publish these notes.

\section{A METHOD OF \\ TREATING DAMAGED INTESTINE WITHOUT RESECTION.}

WITH ILLUSTRATIVE CASES.

By H. BRUNTON ANGUS, M.S., F.R.C.S.,

HONORARY SURGEON, ROYAL VICTORIA INFIRMARY, NEWCASTLEON-TYNE; LECTURER IN SURGERY, UNIVERSITY

$$
\text { OF DURHAM. }
$$

Frequentry when doing emergency work one meets with intestine which is torn, gangrenous, or perforated over alimited area; or, after separating adhesions, free oozing is so persistent as to necessitate immediato treatment.

In many cases of this kind resection appears to be the only resource, but if the patient is old and feeble, exhausted, or suffering from severe shock, time is an im portant consideration, and the following simple method will be found most useful :

Where there is a perforation-for example, from a bullet wound-a suture or two of chromicized catgut passing through all the coats approximates the cdges of the rent; then with Pagenstecher's thread the adjacent sides of the damaged area of intestine are brought together by means of Lembert suture-either continuous or interrupted-thus enclosing the weak spot.

At first sight it appears as though obstruction would result from the kinling of the bowel, but this complication has not ensued after repeated trials.

The method is so simple, and occupies so short a time as comparcd with resection and subsequent anastomosis, that I thought it desirable to publish it, together with one or two examples illustrating its application.

For the drawings illustrating Case II I am indebted to Mr. F. C. Pybus, Surgical Registrar at the Royal Victoria Infirmary, Newcastle-on-Tyne.

CASE I.-Bullet Wounds of the Intestine and Bladder.

A girl, aged 18 years, was admitted into the Royal Victoria Infirmary on June 25th, 1911, after having been shot with a small saloon rifle from behind. The accident occurred at 4 p.m.

On admission (at 5.15 p.m.) there was a small round wound on the left side of the buttock with inverted and slightly burnt elges. The pulse was 80 ; temperature, $98.4^{\circ} \mathrm{F}$

On cxamination there was a little abdominal tenderness and slight abdominal rigidity. Half an hour later she complained of severe abdominal pain, and vomited; the face was pale, the mucous membranes blanched, the pulse 120 , feeble, and poor in volume, the pupils dilated. There was marked abdomina rigidity, with general tenderness. A catheter passed into the bladder evacuated a large quantity of bloody urine. On passing a probe into the wound and with a finger in the rectum, the probe was felt to pass into the pelvis.

Operation (at 6.45 p.m.). - Chloroform and open ether were administered, and a median laparotom below the umbilicus revealed free intestinal contents flooding the whole peritonealcavity. The intestines were examined, and six irregular perforations with protrusion of the mucous membrane were discoveredthree together-in two loops of small intestine, which were lying in the pelvis. The edges of each aperture were drawn
lying tying in the pelvis. The edges of each aperture were drawn together by a single transverse suture passing through all the
coats. The damaged areas were now shut off by suturing all around them, approximating the adjacent surfaces of each loop by means of Pagenstecher's thread. The peritoneal cavity wa flushed with normal sallne at a temperature of $110^{\circ} \mathrm{F}$. No hole was felt or seen in the bladder from the peritoneal aspect, so the abdomen was closed, a large drainage tube inserted.

Subsequent Progress. - There was haematuria for a day or two. The patient had a little chest trouble, and the wound suppurated for a while. She made an excellent recovery.

July 22nd.-She left the hospital well; the scar is stretched.

Readmitted Ausust 29th, and her abdominal wound was sutured in layers on account of commencing hernia. At the same time the bullet was extracted under the skin of the right sime time the bullet was extracted under the skin of the right
side of the pubes. The wounds now healed by first intention, and side of the pubes. The wo
the bowels act normally.

At the second operation the two damaged loops of gut were inspected, and not the slightest trouble was found at the site of operation. The intestinal contents apparently passed through quite freely, the gut on either side of the kink being equally distended.

CASE II.-Bullet Wounds of the Small Intestine.

A young man, aged 25, was admitted into the Royal Victoria Infirmary on October 16th, 1911.

History.-When loading a small air-rifle, with the barrel directed towards his abdomen, the trigger went off and th bullet entered his belly. The accident happened at $10.45 \mathrm{a.m}$.

Operation at 6.30 p.m. (seven and three-quarter hours later) Median laparotomy performed. In the jejunum, $1 \mathrm{ft}$. away f:om the duodeno-jejunal junction, a loop of intestine was

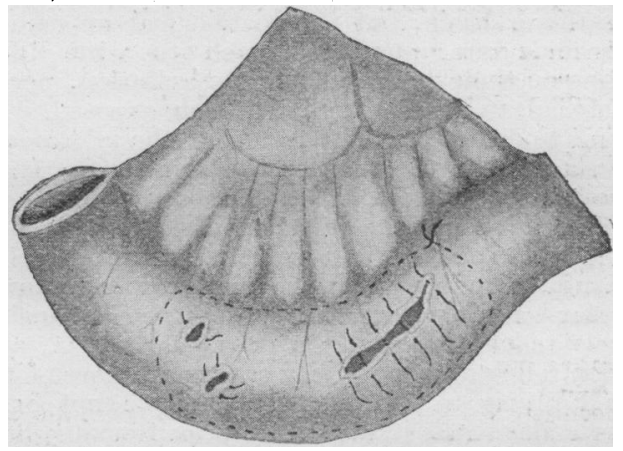

found with two perforations. Two feet away from the duodenojejunal fold another loop of intestine showed three wounds, one jarge and jagged, and two smaller ones. The apertures were large and jagged, and two smaller ones. The apertures were drawn together by sutures passing through all coats, then the damaged areas were covered by approximating the adjacent
surfaces of the bowel by means of Pagenstecher's thread.

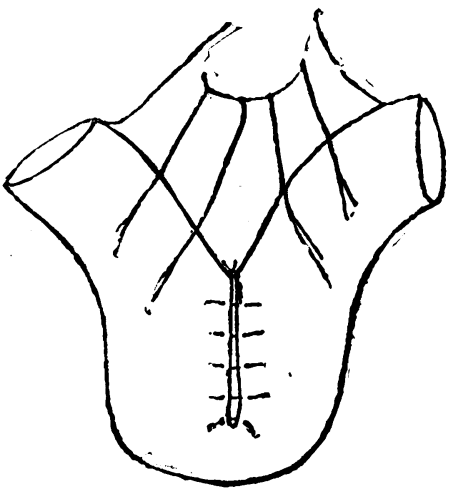

End view of anastomo

These sutures only penetrated peritoneal and muscular coats. The peritoneum was irrigated with normal saline, and the abllomen closed.

Result.-Uninterrupted recovery, bowels act normally. The patient returned seven weeks after the operation in perfect health. The accompanying drawings give a good idea of the nature of the injury, and shows the method of treatment. 